\title{
Neoclassical Viscosities in NCSX and QPS with Few Toroidal Periods and Low Aspect Ratios
}

\author{
Shin NISHIMURA, David R. MIKKELSEN ${ }^{1)}$, Donald A. SPONG ${ }^{2)}$, Steven P. HIRSHMAN ${ }^{2)}$, \\ Long-Poe $\mathrm{KU}^{1)}$, Henry E. MYNICK ${ }^{1)}$ and Michael C. ZARNSTORFF ${ }^{1)}$ \\ National Institute for Fusion Science, 322-6 Oroshi-cho, Toki 509-5292, Japan \\ 1) Princeton Plasma Physics Laboratory, Princeton, New Jersey 08543-0451, USA \\ 2) Oak Ridge National Laboratory, Oak Ridge, Tennessee 37831-6169, USA
}

(Received 16 November 2007 / Accepted 7 February 2008)

\begin{abstract}
Previously reported benchmarking examples of the analytical formulae of neoclassical viscosities were presented implicitly assuming applications in a future integrated simulation system of the Large Helical Device (LHD). Therefore, the assumed toroidal period numbers were mainly $N=10$. However, in this type of calculation, an implicit (or sometimes explicit) assumption of $\iota / N \ll 1$ is sometimes included. This assumption is included not only in simplified bounce-averaged drift kinetic equations for ripple diffusions, but also in the equation before the averaging for non-bounce-averaged effects determining neoclassical parallel viscosity and banana-plateau diffusions. For clarifying the applicability of the analytical methods for configurations with extremely low toroidal period numbers (required for low aspect ratios), we show recent benchmarking examples in the National Compact Stellarator Experiment (NCSX) with $N=3$ and the Quasi-Poloidal Stellarator (QPS) with $N=2$.
\end{abstract}

(C) 2008 The Japan Society of Plasma Science and Nuclear Fusion Research

Keywords: neoclassical transport, neoclassical viscosity, moment equation approach, drift kinetic equation, nonsymmetric toroidal plasma, low aspect ratio stellarator

DOI: $10.1585 /$ pfr.3.S1059

\section{Inroduction}

The moment equation approach for neoclassical transport $[1,2]$ is a powerful method to treat problems in which the field particle portion in the linearized collision term is essentially important. In calculations of the so-called ripple diffusions and their ambipolar condition in collisionless regimes, this portion is not that important. However, the covering area of the neoclassical theory includes various collisionality regimes and also plasma flows. In calculation of the flows, the field particle portion is indispensable in general collisionality regimes, and thus the moment equation approach for non-symmetric toroidal plasmas was developed mainly for neoclassical parallel flows and the associated parallel viscosity [3-6]. Although Sugama and Horton [7] showed that a consistent frame work including both flows and radial diffusions (in other words, not only the parallel viscosity but also poloidal and toroidal viscosities) can be constructed in this line of moment approach, methods for calculating all the required viscosity coefficients in general collisionality regimes in general toroidal configurations had not been shown. Motivated by design activities of advanced stellarators, a method for obtaining the full neoclassical viscosity coefficients was developed in Ref. [8]. It was shown there that three mono-energetic viscosity coefficients $M^{*}$ (parallel viscosity against flows), $N^{*}$ (driving force for bootstrap currents), and $L^{*}$ (radial dif- fusion) are required to describe the full neoclassical characteristics of general non-symmetric toroidal configurations. Since existing numerical methods such as variational and Monte Carlo methods for the drift kinetic equation described in the 3 -D phase space (poloidal angle $\theta$, toroidal angle $\zeta$, pitch angle $\xi$ ) could be applicable [8], the new theory was applied to various types of advanced helical/stellarator configurations $[9,10]$. However, this step of the development of the moment approach was still in the "basic frame work." Although there were many alternative methods for obtaining $L^{*}$ in the collisionless limit (aforementioned ripple diffusions), the other viscosity coefficients could be obtained only by the Drift Kinetic Equation Solver (DKES) code [8-11]. Similar to the MHD equilibrium code, the neoclassical theory should also be a practically usable tool. Faster and easier estimation methods for the neoclassical quantities are desirable in integrated simulation systems using iterative calculations of the equilibrium and transport [12], configuration optimizations [9], and experimental studies investigating dependences on configurations. Since the viscosities (or resulting neoclassical transport coefficients) are direct consequences of guiding center drift motions, evaluating them is important in understanding the characteristics of the designed magnetic configurations [9, 10, 13,14]. Even in tokamak experiments, neoclassical toroidal viscosity effects due to breaking the axisymmetry have recently been 
studied [15], and thus the framework of the moment approach for non-symmetric configurations and the methods for calculating the viscosity coefficients are now required for all studies of toroidal plasma confinements. In theories of axisymmetric tokamaks, simple analytical methods based on asymptotic expansions of the drift kinetic equations and connections of their results are commonly used $[1,16]$. Since this approach using analytical methods will be required in future integrated simulation systems for the Large Helical Device (LHD) [12], we had previously performed derivations and benchmark tests of the analytical formulae for the three mono-energetic coefficients [17].

However, the previous benchmarking examples were made implicitly assuming applications in the LHD (a helical heliotron with major and minor radii of $R_{0}=3.9 \mathrm{~m}$ and $a=0.6 \mathrm{~m}$, respectively, and magnetic field strength of $B_{0} \leq 3 \mathrm{~T}$ ). Therefore, the toroidal period numbers assumed there were mainly $N=10$, and the assumed $B_{m n}^{(\text {Boozer) }}[B=$ $\left.\Sigma B_{m n} \cos (m \theta-n N \zeta)\right]$ spectra did not include $n \neq 0,1$. Although the theory treating the non-bounce-averaged guiding center motion $[3,4]$ is applicable basically for arbitrary $B_{m n}$ spectra, arbitrary aspect ratios, and arbitrary rotational transform per toroidal period $\iota / N$ [17], the benchmarking examples for cases with $n \neq 0,1$, lower aspect ratios, and larger $\iota / N$ had not been reported. A more important open issue is the ripple-trapped/untrapped boundary layer. Although there are many alternative calculating methods for the bounce-averaged motion of the ripple-trapped particles, the boundary layer causes coupling effects between the bounce-averaged motion of ripple-trapped particles and the non-bounce-averaged motion of untrapped particles (collisional detrapping/entrapping). In Ref. [17], a previous boundary layer theory by Shaing and Callen for rippled tokamaks [18] was applied with an extension to multihelicity stellarators. As investigated also in Ref. [17], one effect of this coupling is to make $N^{*}$ (or $G^{(B S)} \equiv$ $\left.-\left\langle B^{2}\right\rangle N^{*} / M^{*}\right)$ in the $1 / v$ regime $\left(E_{s} / v \approx 0\right)$ different from the values given by a theory in Refs. $[3,4]$, which is correct in the collisionless detrapping $v$ regime $\left(E_{s} / v \neq 0\right.$, $v / v \rightarrow 0)$. However, we did not show any benchmarking examples for the $1 / v^{1 / 2}$ diffusion, which is another important effect discussed in Ref. [18]. Even for $N^{*}$ in the $1 / v$ regime, the numerical examples for more general cases had not been shown. For investigating these effects in configurations including $B_{m n}$ of $n \neq 0,1$ and with extremely low toroidal period numbers (required for low aspect ratios) resulting in larger $\iota / N$, we show recent calculation examples in the National Compact Stellarator Experiment (NCSX) [10,13] and the Quasi-Poloidal Stellarator (QPS) $[9,10,14]$ in this paper.

\section{Magnetic Fields in NCSX and QPS}

The NCSX is a quasi-axisymmetric (QA) toroidal system with $N=3, R_{0}=1.4 \mathrm{~m}, a=0.32 \mathrm{~m}$, and $B_{0} \leq 2 \mathrm{~T}$. Figure 1 (a) shows an example of the magnetic field strength
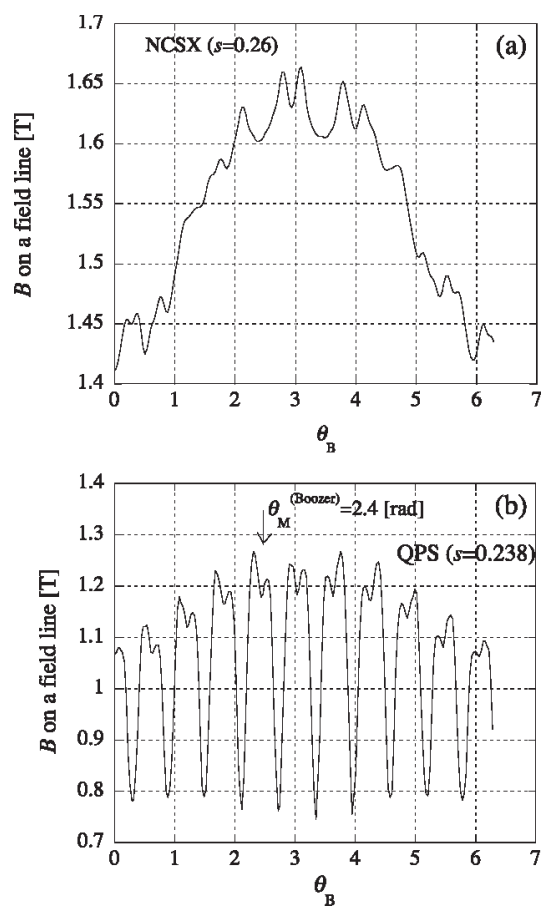

Fig. 1 Magnetic field strengths $B$ on field lines (as functions of the poloidal angle $\theta_{\mathrm{B}}$ in the Boozer coordinates) on flux surfaces with the normalized toroidal flux of $\left(\psi / \psi_{\text {edge }}\right)^{1 / 2} \cong 0.5$ in (a) NCSX and (b) QPS.

on the flux surface in a standard configuration (NCSX$\mathrm{m50)}$ with a finite beta of $\beta=4 \%$ and a finite toroidal current of $I_{p}=178 \mathrm{kA}$. The minor radial position in the figure is that with normalized toroidal flux of $(\psi / \psi \text { edge })^{1 / 2}=0.51$ (corresponding to $r \cong 0.165 \mathrm{~m}$ ). Here the notations for the flux surface coordinates (mainly the Boozer coordinates) in Refs. $[8,17]$ are used, and thus the radial derivatives of the poloidal and toroidal magnetic fluxes are $\chi^{\prime}=0.1178 \mathrm{~T} \cdot \mathrm{m}$ and $\psi^{\prime}=0.2513 \mathrm{~T} \cdot \mathrm{m}$, respectively, and covariant poloidal and toroidal components of the magnetic field are $B_{\theta}=$ $0.0036 \mathrm{~T} \cdot \mathrm{m}$ and $B_{\zeta}=2.3210 \mathrm{~T} \cdot \mathrm{m}$, respectively, on this flux surface. In the numerical examples shown here, we use the flux surface averaged minor radius $r$ as the label of the surfaces $s$, and thus the radial derivatives are denoted by ' $\equiv \mathrm{d} / \mathrm{d} r$. In these calculations in NCSX, the $B_{m n}^{(\text {Boozer) }}$ in a range of $0 \leq m \leq 16$ and $|n| \leq 11$ are used.

The QPS is a quasi-poloidal torus with $N=2, R_{0}=$ $1 \mathrm{~m}, a=0.3 \mathrm{~m}$, and $B_{0} \leq 1 \mathrm{~T}$. As shown in Fig. $1(\mathrm{~b})$, this configuration is designed based on a concept contrasting with QA configurations that reduce the fraction of the ripple-trapped particles. Instead of reducing the fraction, the radial drift of the trapped particle [19] is reduced in this configuration. The parameters of the flux surface in Fig. 1 (b) are $\left(\psi / \psi_{\text {edge }}\right)^{1 / 2}=0.49$ (corresponding to $r \cong$ $0.14 \mathrm{~m}), \chi^{\prime}=0.0275 \mathrm{~T} \cdot \mathrm{m}, \psi^{\prime}=0.1423 \mathrm{~T} \cdot \mathrm{m}, B_{\theta}=0$, and $B_{\zeta}=1.1403 \mathrm{~T} \cdot \mathrm{m}$. In the calculation examples for the QPS in this paper, the $B_{m n}^{\text {(Boozer) }}$ ranges are $0 \leq m \leq 20$ and $|n| \leq 20$.

We shall consider here a modeling method for these 
magnetic fields to estimate analytically the boundary layer effects mentioned in a previous section. As described in Ref. [18], the boundary layer structure is determined by a drift kinetic equation $\left(V_{/ /}-C_{a}^{\mathrm{PAS}}\right) f_{a 1}=0$, where $V_{/ /} \equiv$ $v_{/ /} \mathbf{b} \cdot \nabla_{(\mu=\text { const })}$ is the linearized orbit propagator, and $C_{a}^{\mathrm{PAS}}$ is the pitch-angle-scattering operator with the collision frequency of $v_{\mathrm{D}}^{a}$ [8]. Since $V_{/ /} f_{a 1} \neq 0$ for the non-trivial solution of this equation, the existing bounce- or rippleaveraging methods assuming $V_{/ /} f_{a 1}=0$ are not appropriate for this analysis. Therefore, we use the bounce- or rippleaveraging methods to obtain $\partial f_{a 1} / \partial \mu$ in the ripple-trapped pitch-angle range, which gives the boundary condition for the boundary layer analysis [18], together with the analytical solution for the boundary layer as complimentary methods. For this analytical solution, a model expression of the magnetic field strength $B / B_{0}=1+\varepsilon_{\mathrm{T}}(\theta)+\varepsilon_{\mathrm{H}}(\theta) \cos \{L \theta-$ $N \zeta+\gamma(\theta)\}$ is required. Hereafter, the use of the Boozer coordinates $\left(s, \theta_{\mathrm{B}}, \zeta_{\mathrm{B}}\right)[8]$ is assumed, though we do not write explicitly the subscript "B" indicating "Boozer," since the following analytical approximations on the ripple trapped particles and the boundary layer structure implicitly assume that $\gamma(\theta)$ is a slowly varying function. This characteristic of $\gamma(\theta)$ is not generally satisfied in the Hamada coordinates $\left(s, \theta_{\mathrm{H}}, \zeta_{\mathrm{H}}\right)$. Although a well-known method to obtain this $B$ expression is to truncate $B_{m n}$ with $n \neq$ 0,1 , this truncation is obviously inappropriate for general ripple-trapping $B$ structures such as that in the case in Fig. 1 (b). For trapped particle dynamics and the boundary layer structure, the ripple-well depth is more essential than the detailed ripple-well structure. Therefore, we use $\varepsilon_{\mathrm{H}}(\theta)=\left\{B_{\max }(\theta)-B_{\min }(\theta)\right\} /\left(2 B_{0}\right)$ for each poloidal angle $\theta$ to define $\varepsilon_{\mathrm{H}}(\theta)$. This is a truncation of the Fourier series used by Todoroki [20], who expanded not the amplitude but the phase of $B / B_{0}-1-\varepsilon_{\mathrm{T}}(\theta)$. Similarly, $1+\varepsilon_{\mathrm{T}}(\theta)$ is given by $1+\varepsilon_{\mathrm{T}}(\theta)=\left\{B_{\max }(\theta)+B_{\min }(\theta)\right\} /\left(2 B_{0}\right)$.

In Fig. 1 (a), we can see that the residual ripple-well structure is distorted, or sometimes eliminated at $\theta \approx \pm \pi / 2$ by finite rotational transform per toroidal period $\left(\chi^{\prime} / \psi^{\prime}\right) / N$ in the cases with small $\varepsilon_{\mathrm{H}}$. For such situations, the effective ripple-well depth $\delta_{\text {eff }}$ and length correction $\alpha^{*}$ were introduced in the theory for rippled tokamaks [18]. By extending this method to more general toroidal configurations, we shall define

$$
\begin{aligned}
\delta_{\mathrm{eff}}= & \varepsilon_{\mathrm{H}} \sqrt{1-\alpha^{* 2}} \\
& -\frac{1}{2}\left(\frac{\partial \varepsilon_{\mathrm{T}}}{\partial \theta}+\frac{\partial \varepsilon_{\mathrm{H}}}{\partial \theta} \sqrt{1-\alpha^{*}}\right) \frac{\pi-2 \sin ^{-1} \alpha^{*}}{N \psi^{\prime} / \chi^{\prime}-L-\partial \gamma / \partial \theta}
\end{aligned}
$$

and

$$
\alpha^{*}(\theta)=\frac{\left|\chi^{\prime} \frac{\partial \varepsilon_{\mathrm{T}}}{\partial \theta}\right|}{\sqrt{\left\{\varepsilon_{\mathrm{H}}\left(\chi^{\prime} L-\psi^{\prime} N+\chi^{\prime} \frac{\partial \gamma}{\partial \theta}\right)\right\}^{2}+\left\{\chi^{\prime} \frac{\partial \varepsilon_{\mathrm{H}}}{\partial \theta}\right\}^{2}}} .
$$

The error of a well-known Shaing-Hokin formula [21] for the $1 / v$ ripple diffusions in $\varepsilon_{\mathrm{H}} \rightarrow 0$ limits (e.g., $\varepsilon_{\mathrm{H}} \leq 0.01$ ), which was pointed out in Ref. [8], is strongly reduced by introducing this expression. Using these notations, an expression for the $1 / v^{1 / 2}$ diffusion coefficient in Ref. [18], which is a contribution of ripple-trapped pitch-angle range $0 \leq \kappa^{2} \leq 1$ for $\kappa^{2} \equiv\left\{w-\mu B_{0}\left(1+\varepsilon_{\mathrm{T}}-\delta_{\text {eff }}\right)\right\} /\left(2 \mu B_{0} \delta_{\text {eff }}\right)$, can be extended to a form including more general non-symmetric configurations as

$$
\begin{aligned}
L_{(-1 / 2)}^{*}= & 2.92 \frac{2}{\pi^{2}}\left(\frac{v}{v_{\mathrm{D}}^{a}}\right)^{1 / 2} \frac{2^{3 / 4}}{\left(\psi^{\prime}\right)^{2}}\left(\frac{V^{\prime}}{4 \pi^{2}}\right)^{1 / 2} \\
& \times \int_{-\pi}^{\pi} \frac{\mathrm{d} \theta}{2 \pi} \delta_{\mathrm{eff}}^{3 / 4}\left(B_{0} \frac{\pi-2 \sin ^{-1} \alpha^{*}}{N \psi^{\prime}-L \chi^{\prime}-\chi^{\prime} \partial \gamma / \partial \theta}\right)^{1 / 2} \\
& \times\left\{\left(\frac{\partial \varepsilon_{\mathrm{T}}}{\partial \theta}\right)^{2}-\sqrt{1-\alpha^{* 2}} \frac{\partial \varepsilon_{\mathrm{T}}}{\partial \theta} \frac{\partial \varepsilon_{\mathrm{H}}}{\partial \theta}\right. \\
& \left.+\frac{2}{9}\left(1-\alpha^{* 2}\right)\left(\frac{\partial \varepsilon_{\mathrm{H}}}{\partial \theta}\right)^{2}\right\} .
\end{aligned}
$$

Here, for the aforementioned distribution function in $0 \leq \kappa^{2} \leq 1$ as the boundary condition, an analytical solution by Shaing and Hokin [21],

$$
\begin{aligned}
\frac{\partial G_{X a}^{(1 / v)}}{\partial \mu}= & \frac{c B_{0}}{e_{a} v_{\mathrm{D}}^{a} \psi^{\prime}}\left[\left(\frac{\partial \varepsilon_{\mathrm{T}}}{\partial \theta}+\frac{1}{3} \sqrt{1-\alpha^{* 2}} \frac{\partial \varepsilon_{\mathrm{H}}}{\partial \theta}\right)\right. \\
& \left.-\frac{2}{3} \frac{\kappa^{2} E(\kappa)}{E(\kappa)-\left(1-\kappa^{2}\right) K(\kappa)} \sqrt{1-\alpha^{* 2}} \frac{\partial \varepsilon_{\mathrm{H}}}{\partial \theta}\right] \\
\cong & \frac{c B_{0}}{e_{a} \nu_{\mathrm{D}}^{a} \psi^{\prime}}\left(\frac{\partial \varepsilon_{\mathrm{T}}}{\partial \theta}-\frac{2}{3} \sqrt{1-\alpha^{* 2}} \frac{\partial \varepsilon_{\mathrm{H}}}{\partial \theta}\right)\left(\text { at } \kappa^{2}=0.5\right),
\end{aligned}
$$

$(K(\kappa), E(\kappa)$ : complete elliptic integrals of the first and second kinds), which was also applied in Ref. [17] to derive Eq. (14), is used to consider analytically the dependence on the magnetic configurations, although numerical integral methods [22] also may be applicable for this purpose. From this form of $L_{(-1 / 2)}^{*} \propto \delta_{\text {eff }}^{3 / 4} N^{-1 / 2}$, we can understand that this component of the diffusions can dominate over the $1 / v$ diffusion of $L_{(-1)}^{*} \propto \delta_{\text {eff }}^{3 / 2} N^{0}$ [21] only in configurations with small ripple amplitude $\delta_{\text {eff }}$ and small toroidal period numbers $N$, and therefore it appears in QA configurations rather than the rippled tokamaks considered in Ref. [18]. In fact, previous numerical results in CHS-qa [23] with $N=2$ showed a clear $1 / v^{1 / 2}$ dependence of $L^{*}$ in a wide range of collisionality $(v / v)$. In the next section, we show results in NCSX including the analytical estimation of this effect by Eq. (2). Because of this weak dependence on $\delta_{\text {eff }}$, the analytical estimation of $L_{(-1 / 2)}^{*}$ is still useful even in configurations where numerical integral methods [22] are required for $L_{(-1)}^{*}$. Note that the derivatives $\partial / \partial \theta$ in Eq. (1) must be low-pass-filtered with the cut-off poloidal Fourier mode of $N \psi^{\prime} / \chi^{\prime}-L$, and that in Eqs. (2) and (3) (and also in Eq. (14) in Ref. [17]) must be also filtered with the cut-off mode of $\left(N \psi^{\prime} / \chi^{\prime}-L\right) \pi /\left\langle\pi-2 \sin ^{-1} \alpha^{*}\right\rangle$ in the cases with larger rotational transform per toroidal period $\left(\chi^{\prime} / \psi^{\prime}\right) / N$. Since these derivatives appearing in the analytical solution Eq. (3) expresses the bounce-averaged drift of the ripple-trapped 
particles, contributions of the higher Fourier modes in $\varepsilon_{\mathrm{T}}(\theta)$ and $\varepsilon_{\mathrm{H}}(\theta)$ vanish in the averaging over the bounce period.

\section{Numerical Examples}

Figures 2 (a) and (b) show the mono-energetic viscosity coefficients $M^{*}$ and $N^{*}$ in the NCSX obtained by the analytical formulas [17] and DKES [11]. Following Refs. [8, 17], here we show $G^{(\mathrm{BS})}$ instead of $N^{*}$, and the outputs of the DKES $D_{11}^{*}, D_{13}^{*}$, and $D_{33}^{*}$ are converted to $M^{*}, L^{*}$, and $G^{(\mathrm{BS})} \equiv-\left\langle B^{2}\right\rangle N^{*} / M^{*}$ by Eqs. (54)-(56) in Ref. [8]. The mono-energetic coefficient $L^{*}\left(E_{s} / v \approx 0\right)$ is analytically expressed as the sum of three components: $(1) L_{(-1)}^{*}$ given by appropriate bounce-averaging codes with field line integral methods, (2) $L_{(-1 / 2)}^{*}$ given in Sec. 2, and (3) contributions of non-bounce-averaged drifts given by Eq. (16) in Ref. [17] ( $L_{\text {(banana-plateau) }}^{*}$. We used here the NEO code [22]
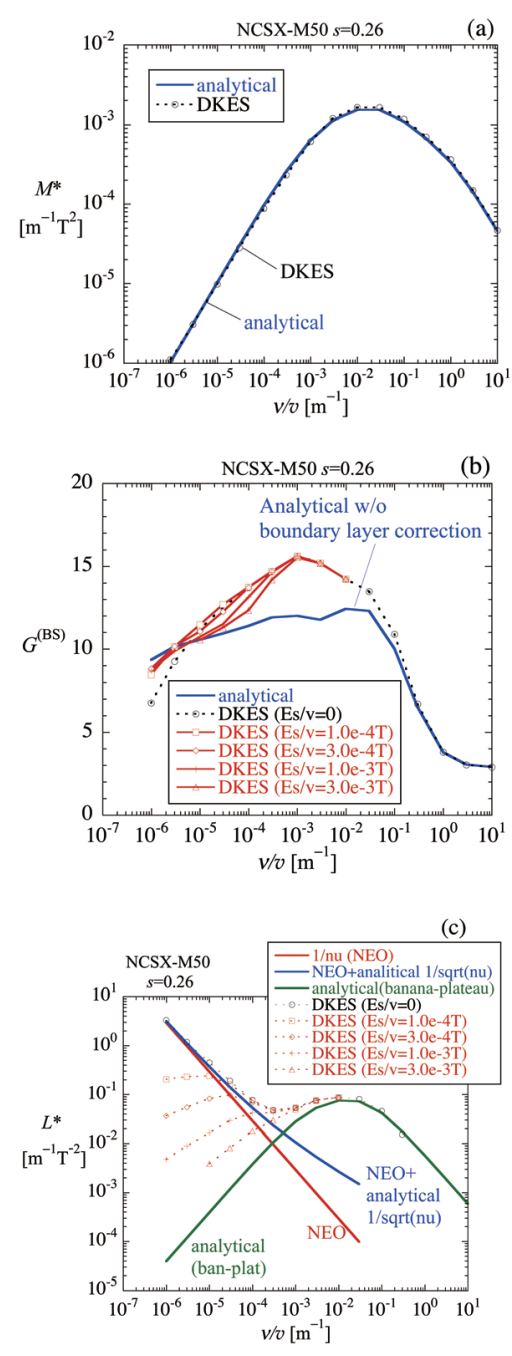

Fig. 2 Mono-energetic viscosity coefficients in the NCSX given by the analytical methods (solid curves) and by the numerical method in the 3-D phase space (DKES) (open symbols). (a) the parallel viscosity $M^{*}$, (b) the geometrical factor $G^{(\mathrm{BS})} \equiv-\left\langle B^{2}\right\rangle N^{*} / M^{*}$, (c) components of the diagonal diffusion $L^{*}$. for the $L_{(-1)}^{*}$ in the NCSX, and Figure 2 (c) shows these components $L_{(-1)}^{*}, L_{(-1)}^{*}+L_{(-1 / 2)}^{*}, L_{(\text {banana-plateau })}^{*}$ and the DKES results. The sum $L_{(-1)}^{*}+L_{(-1 / 2)}^{*}$ approximately predicts a deviation of the DKES from a pure $\propto 1 / v$ scaling given by the bounce-averaging codes at $v / v<10^{-3} \mathrm{~m}^{-1}$. In these figures, we also show the dependences of the DKES results on the $E \times B$ drift parameter $E_{s} / v$. The parallel viscosity coefficient $M^{*}$ is insensitive to the $E \times B$ drift effect in general toroidal configurations [17], and therefore, Fig. 2 (a) shows only the DKES results with $E_{s} / v=$ 0. $N^{*}$ in NCSX is also insensitive to $E_{S} / v$ even in the range of $E_{s} / v \leq 3 \times 10^{-3} \mathrm{~T}$, since the $1 / v$ diffusion of the rippletrapped particles accompanying the boundary layer correction $N_{\text {(boundary) }}^{*}$ in Eq. (14) in Ref. [17] is strongly reduced in this configuration. However, the dependence of the term $L_{(-1)}^{*}+L_{(-1 / 2)}^{*}$ in this $E_{s} / v$ range is not negligible. Although appropriate ripple-averaging methods will be used to calculate this $E \times B$ drift effect on the $L_{(-1)}^{*}+L_{(-1 / 2)}^{*}$ term in the integrated simulation system for the LHD [12], alternative calculation methods applicable for the NCSX with the finite radial electric field is still remaining as a future theme. This problem is discussed in the next section. In spite of this reduction of $L_{(-1)}^{*}$ and accompanied $N_{\text {(boundary), we can }}^{*}$ see another boundary layer effect in Fig. 2 (b). $N^{*}$ given by the DKES transiently becomes larger at $v / v \sim 10^{-3} \mathrm{~m}^{-1}$ compared with the analytical formula. This transient increase is peculiar to QA configurations including the CHSqa [23], where the $1 / v^{1 / 2}$ component becomes comparable or dominates over the $1 / v$ component in the radial diffusion. Although this effect in the $1 / v^{1 / 2}$ regime cannot be calculated by a method in Ref. [17] assuming a collisionless limit of the $1 / v$ regime (the previous formula gives very small values for the QA configurations, and thus is not included in Fig. 2(b)), the transient increase, which is about $30 \%$ at most, will not be so important in the energy integrated coefficients.

Similarly, results in the QPS are shown in Fig. 3. In Fig. 3 (c), the $1 / v$ diffusion coefficient $L_{(-1)}^{*}$ given by the Shaing-Hokin formula [21], including minor modifications of the $B$ expression in Sec. 2, is shown to validate the following discussions on the boundary layer correction, based on Eq. (3). Even for $L_{(-1)}^{*} \propto \delta_{\mathrm{eff}}^{3 / 2}$, the Shaing-Hokin theory Eq. (3) still retains an accuracy of factor 2, in spite of the complex ripple-well structure in Fig. 1 (b). Therefore, we can investigate characteristics of the boundary layer correction on the parallel viscosity $N_{\text {(boundary) }}^{*}$ with a weaker dependence on $\delta_{\text {eff }}$ and $\varepsilon_{\mathrm{H}}$ by the analytical method applying Eq. (3). As confirmed in Ref. [17], we have to interpret a previous " $1 / v$ regime" formula for the parallel viscosity derived by Shaing et al. [3,4] and the $N^{*}$ connection formulae including it (red solid curve in Fig. $3(\mathrm{~b})$ ) as expressions for strong $E_{s} / v$ limit (i.e., the $v$ regime or the $v^{1 / 2}$ regime discussed later) in configurations with large dependences of $L^{*}$ on $E_{s} / v$. The correct $1 / v$ regime $\left(E_{s} / v \approx 0\right)$ value is given by adding a boundary layer correction term $N_{\text {(boundary) }}^{*}$, which was neglected in Refs. [3,4]. 

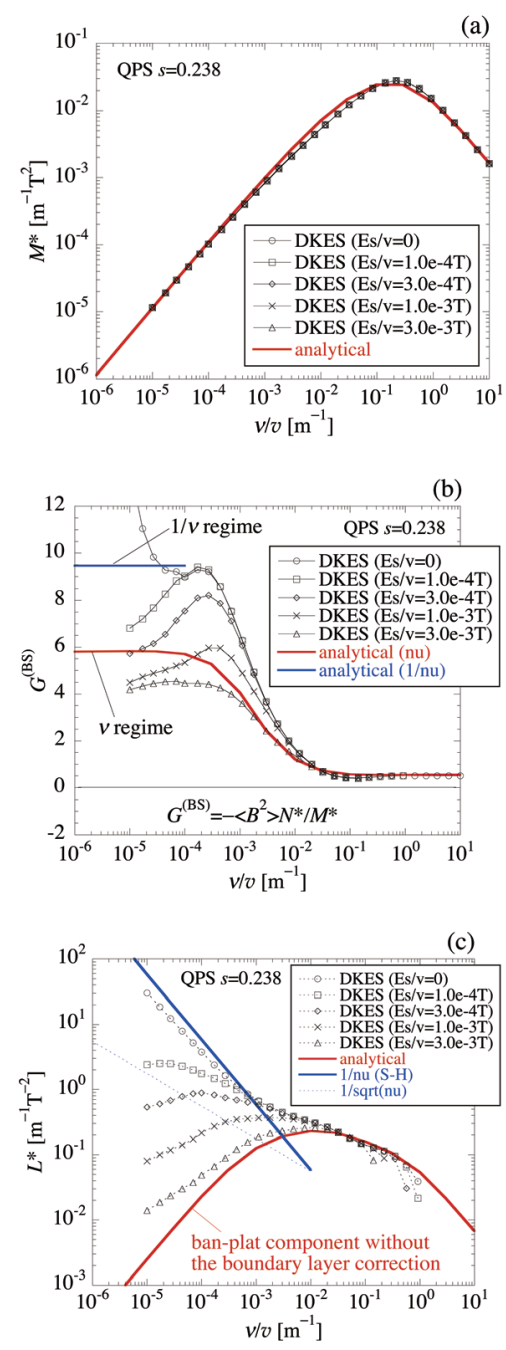

Fig. 3 Mono-energetic viscosity coefficients in the QPS given by the analytical methods (solid curves) and by the DKES (lines with symbols). (a) the parallel viscosity $M^{*}$, (b) the geometrical factor $G^{(\mathrm{BS})} \equiv-\left\langle B^{2}\right\rangle N^{*} / M^{*}$, (c) components of the diagonal diffusion $L^{*}$.

Here, note that the boundary layer theory in Ref. [18] and Eq. (14) in Ref. [17] as its application assumed that the poloidal angle $\theta=\theta_{\mathrm{M}}$ giving maximum value of the magnetic field $\left(B=B_{\mathrm{M}}\right)$, or its envelope Bo $\left\{1+\varepsilon_{\mathrm{T}}(\theta)+\varepsilon_{\mathrm{H}}(\theta)\right\}$ situates $\theta_{\mathrm{M}}= \pm \pi$. We have to extend them to cases with $\pi / 2<\left|\theta_{\mathrm{M}}\right|<\pi$ such as Fig. 1(b) with $\theta_{\mathrm{M}}=$ $\pm 2.4 \mathrm{rad}$, since the boundary condition at the reflection point $v_{/ /}=0$ in Ref. [18] is inverted for the region of $\partial\left(\varepsilon_{\mathrm{T}}+\right.$ $\left.\varepsilon_{\mathrm{H}}\right) / \partial \theta<0$, and resultant $l=1$ moment at $\kappa^{2} \approx 1$ [17] is also inverted. Because of a small fraction of toroidally trapped particles compared with a large fraction of rippletrapped particles in the QPS (i.e., $\partial\left(\varepsilon_{\mathrm{T}}+\varepsilon_{\mathrm{H}}\right) / \partial \theta \ll \varepsilon_{\mathrm{H}}$ ), another minor modification to Ref. [17] for the QPS is replacing $\left[\partial G_{X a}^{(1 / v)} / \partial \mu\right]_{\kappa^{2}=0.5}$ by $\left[\partial G_{X a}^{(1 / v)} / \partial \mu\right]_{\kappa^{2}=1.0}$. The existence of the collisional perturbation function component in the toroidally trapped pitch-angle-range of $w / B_{\mathrm{M}} \leq \mu<$ $w /\left\{B_{0}\left(1+\varepsilon_{\mathrm{T}}+\delta_{\text {eff }}\right)\right\}$ with axisymmetric Fourier mode $n=0$, which remains even in the $1 / v$ regime, is restricted in very narrow $\mu$ range, and thus tends to be determined by the bounce-averaged part only in $\mu \approx w /\left\{B_{0}\left(1+\varepsilon_{\mathrm{T}}+\delta_{\text {eff }}\right)\right\}$. By including these modifications, $N_{\text {(boundary) }}^{*}$ in the QPS configuration is estimated by

$$
\begin{aligned}
& N_{\text {(boundary) }}^{*}=-\frac{12}{\pi^{3}} \frac{v_{\mathrm{D}}^{a}}{v} \frac{\left\langle B^{2}\right\rangle}{\chi^{\prime} \psi^{\prime} f_{\mathrm{c}}} \frac{V^{\prime}}{4 \pi^{2}} \\
& \times\left[\int_{0}^{\theta_{\mathrm{M}}} \mathrm{d} \theta\left(2 \delta_{\text {eff }}\right)^{1 / 2}\left(\pi-2 \sin ^{-1} \alpha^{*}\right) \theta\left(\frac{\partial \varepsilon_{\mathrm{T}}}{\partial \theta}-\frac{1}{3} \sqrt{1-\alpha^{* 2}} \frac{\partial \varepsilon_{\mathrm{H}}}{\partial \theta}\right)\right. \\
& \left.-\int_{\theta_{\mathrm{M}}}^{\pi} \mathrm{d} \theta\left(2 \delta_{\text {eff }}\right)^{1 / 2}\left(\pi-2 \sin ^{-1} \alpha^{*}\right) \theta\left(\frac{\partial \varepsilon_{\mathrm{T}}}{\partial \theta}-\frac{1}{3} \sqrt{1-\alpha^{* 2}} \frac{\partial \varepsilon_{\mathrm{H}}}{\partial \theta}\right)\right] .
\end{aligned}
$$

Although more general discussions about the region of $\partial\left(\varepsilon_{\mathrm{T}}+\varepsilon_{\mathrm{H}}\right) / \partial \theta \leq 0$ in $0<\theta<\pi$ are complicated [17] and will be discussed elsewhere, this region is small in practically important configurations with well-performed drift optimizations [19], and thus these complicated discussions are not important. In Fig. 3 (b), we showed the $1 / v$ regime asymptotic value of $N^{*}$ given by $N^{*(\text { sym })}+N^{*(\text { asym })}+$ $N_{\text {(boundary) }}^{*}$ [17] with Eq. (4). It approximates the numerical result for a weak radial electric range of $E_{S} / v<1 \times 10^{-4} \mathrm{~T}$ by the DKES. Therefore the dependence of the coefficient $N^{*}$ (or $D_{13}$ ) on the radial electric field, which was commonly found in non-axisymmetric toroidal configurations, can be interpreted as a suppression of the $\propto 1 / v$ component of the perturbation Eq. (3) by the $E \times B$ drift. In $E_{s} / v$ ranges without the toroidal resonance [3] (one reason for which we restrict here this range to be $E_{s} / v \leq 3 \times 10^{-3} \mathrm{~T}$ ), the $E \times B$ drift effect on the ripple-untrapped particles in $\kappa^{2}>1$ is not important because of $V_{/ /} f_{a 1} \neq 0$.

Nevertheless, we can see in Fig. 3 (b) another problem, which is peculiar to configurations with extremely small toroidal period numbers. The DKES result with $E_{s} / v=0$ begins to diverge to a larger value without converging to an asymptotic value in $v / v<10^{-4} \mathrm{~m}^{-1}$. This behavior of $N^{*}$ (or $D_{13}$ ) in extremely collisionless limits with weak radial electric fields $\left(v / v, E_{s} / v \rightarrow 0\right)$ often appears in general configurations (analogous behavior can be seen also at $v / v \leq 10^{-6} \mathrm{~m}^{-1}$ in Fig. 2) because of a singularity of $\propto\left(\chi^{\prime} m-\psi^{\prime} N n\right)^{-1}$ in $N^{*(\text { asym })}[3,4,17]$. Sometimes it appears even in shorter mean free path range in configurations with small $N$. One reason for a deviation of the connection formula (red solid curve) from the DKES result with $E_{s} / v=1 \times 10^{-3} \mathrm{~T}, 3 \times 10^{-3} \mathrm{~T}$ and $v / v<10^{-4} \mathrm{~m}^{-1}$ of about $30 \%$ is that the analytical formula includes terms with $\chi^{\prime} m-\psi^{\prime} N n \approx 0$ in spite of a fact that these terms are actually suppressed by finite $E \times B$ drifts. Even in cases with $E_{s} / v \approx 0$, these terms will be suppressed by higher order magnetic drifts and collision effects; therefore, actually, the Fourier series of $N^{*(\text { asym) }}$ must be appropriately truncated, or suppression of these resonant terms have to be included in the series. It should also be noted regarding Fig. 3 (c) that the "analytical" $L^{*}$ is given by a formula in Sec.V in Ref. [17] neglecting the boundary layer effects on the ripple-untrapped pitch-angle range $\kappa^{2}>1$. How- 
ever, there is a non-negligible effect on $L^{*}$ near the collisionality regime boundary between the banana $(1 / v)$ and plateau regimes. This effect is analogous to the increase of $N^{*}$ in $10^{-3} \mathrm{~m}^{-3}<v / v<10^{-2} \mathrm{~m}^{-3}$ and with $E_{s} / v \approx 0$ by $N_{\text {(boundary) }}^{*}$, and makes $L^{*}$ in this $\left(v / v, E_{s} / v\right)$ range transiently larger than the present analytical calculation even when a correct $1 / v$ diffusion coefficient is added. It is a contribution of the ripple-untrapped pitch-angle range $\kappa^{2}>1$, which differs from that of $0 \leq \kappa^{2} \leq 1$ expressed in Eq. (2) Because of the dependences of $L_{(-1 / 2)}^{*}$ and $L_{(-1)}^{*}$ on $\delta_{\text {eff }}$ discussed in the previous section, this equation gives a too small value in the QPS to explain the $L^{*}$ in $10^{-3} \mathrm{~m}^{-3}$ $<v / v<10^{-2} \mathrm{~m}^{-3}$. This approximation of $L^{*}$ in configurations without quasi-axisymmetry can be improved by adding an appropriate connection formula for the contribution of the ripple-untrapped particle distribution $L_{\text {(boundary) }}^{*}$ [17].

\section{Concluding Remarks}

The mono-energetic neoclassical viscosity coefficients are investigated in two low-aspect stellarator configurations with contrasting design concepts. For $M^{*}$, $N^{*(\text { asym) }}$, and $\left(\sigma_{X a}, G_{X a}^{(\text {asym) }}\right)$ defined in Ref. [17] due to pure non-bounce-averaged motions $[3,4]$, the validity of the analytically approximated formulas $[8,17]$ has been confirmed even in the NCSX and the QPS. The formulae for these components are basically applicable for general toroidal configurations except that two minor modifications are required there relating to resonant Fourier modes in $N^{*(\text { asym) }}$ and the integration constant in the banana regime expansion of $\left(V_{/ /}-G_{a}^{\mathrm{PAS}}\right) G_{X a}^{(\text {asym })}=\sigma_{X a}^{(\text {asym) }}$ as discussed in Sec. 2 in Ref. [17].

In the two configurations, there are contrasting effects of the ripple-trapped/untrapped boundary layer at $\kappa^{2} \cong$ 1 causing coupling effects between the bounce-averaged motion of ripple-trapped particles and the non-bounceaveraged motion of untrapped particles. The $1 / v^{1 / 2}$ ripple diffusion $L_{(-1 / 2)}^{*}$ in the QA configurations is peculiar to the configurations with small ripples. In the ripple-trapped pitch angle range $0 \leq \kappa^{2} \leq 1$ in these configurations, the $1 / v^{1 / 2}$ component of the perturbed distribution function as an integration constant for Eq. (3) determined by the boundary layer [18] is not negligible compared with the small $1 / v$ component. However, their effects on the rippleuntrapped pitch-angle range $\kappa^{2}>1$ are not important. In contrast to this, the boundary layer affects the range $\kappa^{2}>1$ in configurations without quasi-axisymmetry, and makes other boundary layer corrections on the viscosity coefficients; $N_{\text {(boundary) }}^{*}$ appearing in the $1 / v$ regime $\left(E_{s} / v \approx 0\right)$ and also $L_{\text {(boundary) }}^{*}$ near the collisionality regime boundary between $1 / v$ and plateau regimes. Although the integration constant in $0 \leq \kappa^{2} \leq 1$ is negligible compared with a large $1 / v$ component, boundary layer effects as a driving force of $\propto\left(\delta_{\text {eff }}\right)^{1 / 2}$ for the flows in $\kappa^{2}>1$ is not negligible for the $\propto v^{0}$ component of the distribution function in these con- figurations without quasi-axisymmetry. Although we analyzed these effects by applying a simple analytical theory (Eq. (3)) for the trapped range of $0 \leq \kappa^{2} \leq 1$, the other numerical integral methods [22] and more accurate analytical methods [20] retaining higher orders of $\varepsilon_{\mathrm{T}}, \varepsilon_{\mathrm{H}}$, and higher modes in the "phase" Fourier series of $B / B_{0}-1-\varepsilon_{\mathrm{T}}(\theta)$ will be applicable for this purpose.

We concentrated mainly on cases of $E_{s} / v \approx 0$ in this paper, and thus it also should be noted that remaining future themes are in finite radial electric field effects. In “drift-optimized" configurations (not only NCSX and QPS but also the inward shifted configurations in the heliotrons, which is an application of an idea of " $\sigma$-optimization" [19]), the $v^{1 / 2}$ regime ripple diffusion appears in wide ranges of the collisionality in numerically obtained $L^{*}$ for $E_{s} / v \neq 0$. In other words, the $v^{1 / 2}$ regime diffusion is also reduced by the optimizations compared with the so-called single helicity model $B / B_{0}=1-\varepsilon_{\mathrm{t}} \cos \theta+\varepsilon_{\mathrm{h}} \cos (L \theta-$ $N \zeta)$ in Refs. [8, 17]. Although a mechanism of this reduction is qualitatively an enhanced collisionless entrapping/detrapping, it had not been described quantitatively by conventional analytical theories. In contrast to many detailed studies on the dependence of the $1 / v$ diffusion on $B_{m n}$ spectra using various methods [21,22], the dependence of the $v^{1 / 2}$ diffusion had not been investigated in detail. One complexity makes the analysis difficult is the fact that this is another "boundary layer problem" with $E_{s} / v \neq 0$, which differs from the boundary layer problem with $E_{s} / v=$ 0 discussed in this paper. To understand this $v^{1 / 2}$ diffusion is not only useful for the reduction of the computational efforts for $L^{*}$, but also is important in making the connection formula of $N^{*}$ in $1 / v, v^{1 / 2}$, and $v$ regimes. A previous example of this connection formula in Ref. [17] using an empirical scaling given by E. C. Crume, Jr., et al. [24], who neglected this $v^{1 / 2}$ regime term in their expression for $L^{*}$, is not appropriate in these optimized cases. Although we did not discuss on this problem in this paper, it also is now under study to complete the analytical calculation of $N^{*}$ in intermediate $E_{s} / v$ ranges in Fig. 3 (b).

\section{Acknowledgements}

A main part of this work was done during one author (S.N.)'s visit to the Princeton Plasma Physics Laboratory (PPPL) and the Oak Ridge National Laboratory (ORNL), which was sponsored by the Graduate University for Advanced Studies (GUAS). The authors thank Dr. Shunsuke Usami in the National Institute for Fusion Science (NIFS) for his help in installing the ORNL library files for the DKES into NEC SX-8 in NIFS. The one author (S.N.) thanks also the director-general Osamu Motojima in NIFS for the encouragements for this work of GUAS. A NIFS coordinated research program NIFS07KNXN103 also supported this study.

[1] S.P. Hirshman and D.J. Sigmar, Nucl. Fusion 21, 1079 
(1981).

[2] K.C. Shaing and J.D. Callen, Phys. Fluids 26, 3315 (1983).

[3] K.C. Shaing, E.C. Crume, Jr. et al., Phys. Fluids B1, 148(1989).

[4] K.C. Shaing, B.A. Carreras et al., Phys. Fluids B1, 1663(1989).

[5] N. Nakajima and M. Okamoto, J. Phys. Soc. Jpn. 61, 833 (1992).

[6] N. Nakajima, M. Okamoto and M. Fujiwara, J. Plasma Fusion Res. 68, 46 (1992).

[7] H. Sugama and W. Horton, Phys. Plasmas 3, 304 (1996).

[8] H. Sugama and S. Nishimura, Phys. Plasmas 9, 4637 (2002).

[9] D.J. Strickler, S.P. Hirshman, D.A. Spong et al., Fusion Sci. Technol. 45, 15 (2004).

[10] D.A. Spong, Phys. Plasmas 12, 056114 (2005).

[11] W.I. van Rij and S.P. Hirshman, Phys. Fluids B1, 563 (1989).

[12] Y. Nakamura, M. Yokoyama, N. Nakajima et al., Fusion Sci. Technol. 50, 457 (2006).

[13] D.R. Mikkelsen, H. Maassberg, M.C. Zarnstorff et al., Fusion Sci. Technol. 51, 166 (2007); G.H. Neilson, J.F. Lyon, M.C. Zarnstorff et al., in $16^{\text {th }}$ International Stellarator
Workshop Oct.15-19, 2007, Toki, Japan O-13.

[14] D.A. Spong, S.P. Hirshman et al., Nucl. Fusion 45, 918 (2005); J.F. Lyon, B.E. Nelson, J.H. Harris et al., in $16^{\text {th }}$ International Stellarator Workshop Oct.15-19, 2007, Toki, Japan P2-095.

[15] W. Zhu, S.A. Sabbagh et al., Phys. Rev. Lett. 96, 22002 (2006).

[16] W.A. Houlberg, K.C. Shaing, S.P. Hirshman and M.C. Zarnstorff, Phys. Plasmas 4, 3230 (1997).

[17] S. Nishimura, H. Sugama and Y. Nakamura, Fusion Sci. Technol. 51, 61 (2007).

[18] K.C. Shaing and J.D. Callen, Phys. Fluids 25, 1012 (1982).

[19] H.E. Mynick, Phys. Fluids 26, 1008 (1983).

[20] J. Todoroki, J. Phys. Soc. Jpn. 8, 2758 (1990).

[21] K.C. Shaing and S.A. Hokin, Phys. Fluids 26, 2136 (1983).

[22] V.V. Nemov, S.V. Kasilov, W. Kernbichler and M.F. Heyn, Phys. Plasmas 6, 4622 (1999).

[23] S. Okamura, K. Matsuoka et al., Nucl. Fusion 44, 575 (2004).

[24] E.C. Crume, Jr., K.C. Shaing, S.P. Hirshman and W.I. van Rij, Phys. Fluids 31, 11 (1988). 